\title{
Calidad de Vida y Apoyo Social de los Pacientes con Enfermedad Pulmonar Obstructiva Crónica
}

\author{
Quality of life and social support for patients suffering from chronic \\ obstructive pulmonary disease
}

Robinson Ramírez-Vélez

Fundación Universitaria Maria Cano, Cali. robin640@hotmail.com

Recibido 26 Enero 2007/Enviado para Modificación 23 Septiembre 2007/Aceptado 20 Octubre 2007-10-20

RESUMEN

Objetivo Describir en los pacientes con Enfermedad Pulmonar Obstructiva Crónica la calidad de vida y el apoyo social con que cuentan.

Métodos Estudio descriptivo, trasversal $(n=22)$ sujetos que obedecieron a los criterios de inclusión. La salud calidad de vida se determinó a través del Cuestionario de Salud Nottingham Health Profile (NHP), y el apoyo social con la (Escala de DukeUnc).

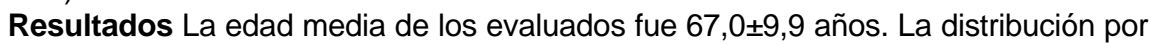
género fue 8 hombres 36,4 \% y 14 mujeres 63,6 \%. Las puntuaciones más altas de calidad de vida se reflejaron en los dominios Sueño $42,3 \pm 30,8$, Reacciones Emocionales $40,2 \pm 5,7$ y Movilidad 38,4 $\pm 33,5$, mientras que la puntuación más baja se demostró en el dominio Aislamiento Social con 23,6 $\pm 19,4$. El Apoyo Social Afectivo bueno: 55,1 \% de pacientes, Apoyo Social Confidencial escaso: 44,9\% de pacientes. Conclusiones Las mujeres muestran una percepción peor de su estado de salud y calidad de vida que los hombres principalmente en los dominios Sueño, Reacciones Emocionales, Energía y Movilidad. Algo más de la mitad de los pacientes referían buen apoyo social, siendo mayor el afectivo que el confidencial.

Palabras Clave: Enfermedad Pulmonar Obstructiva Crónica, calidad de vida, apoyo social (fuente: DeCS, BIREME).

\section{ABSTRACT}

Objective Describing patients suffering from chronic obstructive pulmonary disease, their quality of life and their respective social support network.

Methods This was a cross-sectional study of patients $(n=22)$ fulfilling the inclusion criterion. Quality of life regarding health was determined by using the Nottingham Health Profile (NHP) and the Duke-UNC scale for the social support network.

Results The patients' average age was $67,0 \pm 9,9$. Gender distribution was 8 male $(36,4 \%)$ and 14 female patients $(63,6 \%)$. The highest point-scores for quality of life on the Nottingham Health Profile subscales were obtained for sleep $(42,3 \pm 30,8)$, 
emotional reactions $(40,2 \pm 25,7)$ and mobility $(38,4 \pm 33,5)$ whilst the lowest pointscore was obtained on the social isolation subscale $(23,6 \pm 19,4)$. There was good emotional social support $(55,1 \%$ of patients) but limited confidential social support (44,9\% of patients).

Conclusions Women showed worse perception regarding their state of health and quality of life than men. The following dimensions being mostly affected: sleep, emotional reactions, energy and mobility. Just over half the patients referred to having acceptable social support; emotional support was greater than confidential support.

Key Words: Chronic Obstructive Pulmonary Disease, health-related quality of life, social support (source: MeSH, NLM).

$\mathrm{E}$ n el año 2020 la Enfermedad Pulmonar Obstructiva Crónica (EPOC) será la tercera causa de muerte en todo el mundo (1). Este proceso se caracteriza por una limitación apenas reversible del flujo respiratorio y la disnea. A medida que la enfermedad progresa, algunos pacientes desarrollan manifestaciones sistémicas, incluida una limitación del ejercicio, disfunción muscular periférica, hipertensión pulmonar, marcada desnutrición, y exacerbaciones recurrentes que dan lugar a hospitalizaciones $(2,3)$. Por esta razón, la evaluación y el tratamiento de esta enfermedad partiendo exclusivamente de la limitación del flujo respiratorio niegan la importancia de otras manifestaciones clínicas tratables del proceso. A medida que esta entidad clínica progresa, los pacientes experimentan una disminución de la calidad de vida relacionada con la salud (CVRS), con empeoramiento de su capacidad para trabajar y disminución en la participación de actividades físicas y sociales lo que repercute directamente en su estilo de vida, de acuerdo con su propia percepción e interpretación. Este aspecto subjetivo de la morbilidad (gravedad percibida por el paciente) es lo que definimos como (CVRS), término que se ha convertido en los últimos años en objetivo importante a ser alcanzado en diferentes intervenciones en salud $(4,5)$ reforzando el consenso que los indicadores utilizados tradicionalmente como el control de los síntomas, índices de mortalidad y la expectativa de vida, no bastan, por si solos, como estimaciones de la eficacia y efectividad de los programas e intervenciones introducidas para evaluar muchos de los tratamientos en estos pacientes (6). Adicionalmente, el tener un buen apoyo social, entendido como el grado en el que las necesidades sociales básicas son satisfechas, puede favorecer el manejo de la intervención preventiva, terapéutica y rehabilitadora (6). Partiendo de estas asociaciones, el presente estudio tuvo como propósito describir "cómo se encuentran desde el punto de vista psicosocial" valorando para ello, su calidad de vida (salud autopercibida) y su apoyo social en una muestra de pacientes con EPOC 
REVISTADE SALUD PÚBLICA•Volumen 9 (4), Diciembre 2007

atendidos por el servicio de Fisioterapia de la Unidad hospitalaria Clínica Rafael Uribe Uribe Empresa Social del Estado Antonio Nariño. Cali, 2006.

\section{MÉTODOS}

Mediante un estudio descriptivo, transversal, fueron incluidos 101 pacientes del servicio de Medicina Interna y Urgencias de la Unidad Hospitalaria Clínica Rafael Uribe Uribe, ESE Antonio Nariño de la ciudad de Cali. Posteriormente mediante un muestreo sistemático, fueron escogidos los pacientes que cumplieron a los criterios de pre-selección (enfermedades del sistema respiratorio) entre el 1 de septiembre y el 1 de noviembre de 2006. Como criterios de Inclusión se tuvieron en cuenta los sujetos con diagnóstico clínico y/o espirométrico de EPOC, facultativo por el personal especializado y distinguido mediante Código Único de Procedimientos en Salud (CUPS), quienes contaban con su historia clínica dentro la Unidad Hospitalaria, de ambos sexos, con edad superior a 35 años y hallan aceptado su participación otorgado su consentimiento libre informado por escrito. Se excluyeron los pacientes con diagnóstico de asma, neumonía, bronquiectasias, o tuberculosis, entidades explícitamente excluidas por la SEPAR (6) y por la GOLD (5-7) de la definición de EPOC. Se consideró paciente diagnosticado de EPOC cuando aparecía de forma explícita este diagnóstico (CUPS), en la historia clínica de los pacientes. Al final la población muestra que cumplieron a los criterios de inclusión fueron 22 sujetos. Las variables recogidas objeto de este estudio fueron: Variables Sociodemográficas: edad, género, estado civil, estrato socioeconómico. Variables antropométricas y estado nutricional: peso corporal y talla, las cuales fueron valoradas siguiendo las normas dictadas y establecidas por el ISAK (8) y GREC (9). Con estas dos variables se determino el Índice de Masa Corporal (IMC), según Harmon (10). La CVRS: fue evaluada por medio del cuestionario de salud NHP (11) para medir la percepción subjetiva y el impacto en los problemas de salud $(12,13)$. Cada sesión contiene preguntas que son registradas y convertidas en scores que van del 0 al 100, donde 0 es mejor percepción de salud y 100 refiere peor estado de salud $(13,15)$. El estudio aplicó la versión ajustada NHP validado en castellano por Alonso (11-12). Por último, se preguntó por el apoyo social: a través de la escala de DUKE-UNC (14), que consta de 11 preguntas, la cual busca los valores referidos al apoyo afectivo y confidencial (14). El estudio aplico el cuestionario validado por Alvarado B, en el 2005(15). El procesamiento de los datos se realizo calculando estadísticos descriptivos para las variables cuantitativas. También fueron calculados los IC 95\%. El nivel de significancia aceptado fue $\mathrm{p}=<0.05$, y el análisis de las variables se hizo en el programa Excel, para Windows XP 2002 y SPSS versión 12. 


\section{RESULTADOS}

Variables Sociodemográficas. La edad media de la muestra fue 67,0 $\pm 9,9$ años (IC 95 \% 66,7-68,1), en un rango de 35 a 80 años. El 45,5 \% tenían una edad comprendida entre 61 y 70 años. La distribución general por género que fue de 8 hombres y 14 mujeres (Tabla 1 ).

Antropometría y estado nutricional. El peso corporal medio fue de $63.0 \pm 9.9$

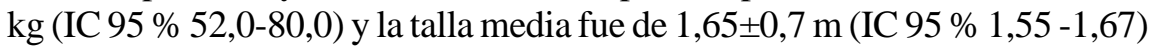
y el IMC de 23,7 $\pm 4,6 \mathrm{~kg} / \mathrm{m} 2$ (IC $95 \% 22,0-26,0$ ).

Tabla 1. Características sociodemográficas de la Población

\begin{tabular}{lccc}
$\begin{array}{c}\text { Variables } \\
\text { Sociodemográficas }\end{array}$ & $\begin{array}{c}\text { Frecuencia } \\
\text { relativa } \\
\mathrm{n=22}\end{array}$ & $\begin{array}{c}\text { Frecuencia } \\
\text { absoluta (\%) } \\
100\end{array}$ & $\begin{array}{c}\text { Intervalos } \\
\text { de confianza } \\
\text { (IC) }\end{array}$ \\
\hline Edad & 1 & 4,5 & $0,29-10,9$ \\
Menos de 40 años & 3 & 13,6 & $11,4-16,9$ \\
$41-50$ años & 5 & 22,7 & $21,9-41,2$ \\
$51-60$ años & 10 & 45,5 & $35,7-55,2$ \\
$61-70$ años & 3 & 13,6 & $2,21-19,9$ \\
\hline Más de 71 años & & & \\
\hline Género & 8 & 37,4 & $30,9-42,1$ \\
Hombres & 14 & 63,6 & $55,6-73,7$ \\
Mujeres & & & \\
\hline Estado Civil & 6 & 27,3 & $-9,7-33,5$ \\
Soltero & 9 & 40,9 & $3,5-45,2$ \\
Casado & 4 & 18,2 & $4,8-27,8$ \\
Unión Libre & 2 & 9,1 & $-9,7-13,5$ \\
Separado & 1 & 4,5 & $0,27-12,5$ \\
Viudo & & & \\
\hline Estrato Social & 6 & 27,3 & $9,5-33,9$ \\
I & 4 & 18,2 & $11,5-29,2$ \\
II & 10 & 45,5 & $39,5-48,7$ \\
III & 2 & 9,1 & $0,65-11,3$ \\
IV & 0 & 0,0 & - \\
V & 0 & 0,0 & - \\
VI & & &
\end{tabular}

Tabla 2. Puntuaciones generales del Cuestionario de Salud NPH y Cuestionario de Apoyo Social (Escala de Duke-Unc) de la Población.

\begin{tabular}{|c|c|c|}
\hline Dominios & Mediana/DE & Intervalos de confianza (IC 95\%) \\
\hline Energia & $36,3 \pm 44,7$ & $23,3-55,6^{*}$ \\
\hline Dolor & $34,2 \pm 36,3$ & $32,1-42,2$ \\
\hline Reacciones Emocionales & $40,2 \pm 25,7$ & $39,5-38,4^{*}$ \\
\hline Sueño & $42,3 \pm 30,8$ & $37,9-45,7^{*}$ \\
\hline Aislamiento Social & $23,6 \pm 19,4$ & $19,3-27,9$ \\
\hline Movilidad & $38,4 \pm 33,5$ & $36,7-43,1$ \\
\hline Total & $40,7 \pm 23,9$ & $38,3-55,2$ \\
\hline \multicolumn{3}{|c|}{ Puntuación Apoyo Social (Escala de Duke-Unc) } \\
\hline APOYO AFE & $12,3 \pm 14,7$ & $6,0-30,0$ \\
\hline APOYO CON ${ }^{b}$ & $25,2 \pm 16,3$ & $5,0-25,0$ \\
\hline APOYO SOC & $40,8 \pm 15,7$ & $11,0-55,0$ \\
\hline
\end{tabular}

DE: desviación estándar; * $\mathrm{P}<0.05$; $\mathrm{a}$. Apoyo Social Afectivo bueno si la puntuación media es mayor de 18 puntos; b. Apoyo Social Confidencial bueno si la puntuación media es mayor de 15 puntos; c. Apoyo Social total bueno si la puntuación media es mayor de 33 puntos. 
REVISTADE SALUD PÚBLICA · Volumen 9(4), Diciembre 2007

Calidad de vida. En la Tabla 2, se presentan las puntuaciones del Cuestionario de Salud NPH. En general el Sueño es la dimensión que obtiene una mayor

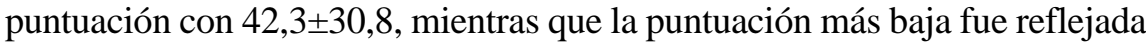
en el Aislamiento social 23,6 \pm 19,4. La puntuación total del NPH fue de 40,7 $\pm 23,9$. En la Figura 1, se presentan las calificaciones obtenidas entre géneros (enunciado en mediana), de todos los dominios del NHP. Las puntuaciones más elevadas referidas por los hombres se hallaron en el dominio Sueño 42,3 $\pm 24,3$, seguido de Reacciones Emocionales 27,5 $\pm 22,3$, mientras que la puntuación más baja se reflejó en la categoría Energía 00,0 035,3. En cuanto a las mujeres evaluadas, llama la atención la variabilidad encontrada y las puntuaciones elevadas (mayores del $50 \%$ ) en el dominio Energía 66,0 $\pm 48,1$, seguido de Movilidad 62,3 $\pm 22,3$, mientras que la puntuación más baja fue en la categoría Aislamiento Social 35,0 $\pm 19,8, \mathrm{p}=<0.05$.

Apoyo Social. En la Tabla 2, se presentan los resultados del apoyo social. La puntuación media del apoyo social afectivo fue de 12,3 (bueno $>18$ puntos) y del apoyo social confidencial fue de 25,2 (bueno $>15$ puntos). El 55,1 \% de los pacientes referían tener un buen apoyo social de forma global (>33 puntos), siendo mayor el porcentaje de pacientes que presentaban un buen apoyo afectivo (55,1 \%) que el confidencial (44,9\%).

Figura 1. Comparación por géneros de las puntuaciones del Cuestionario de Salud NPH

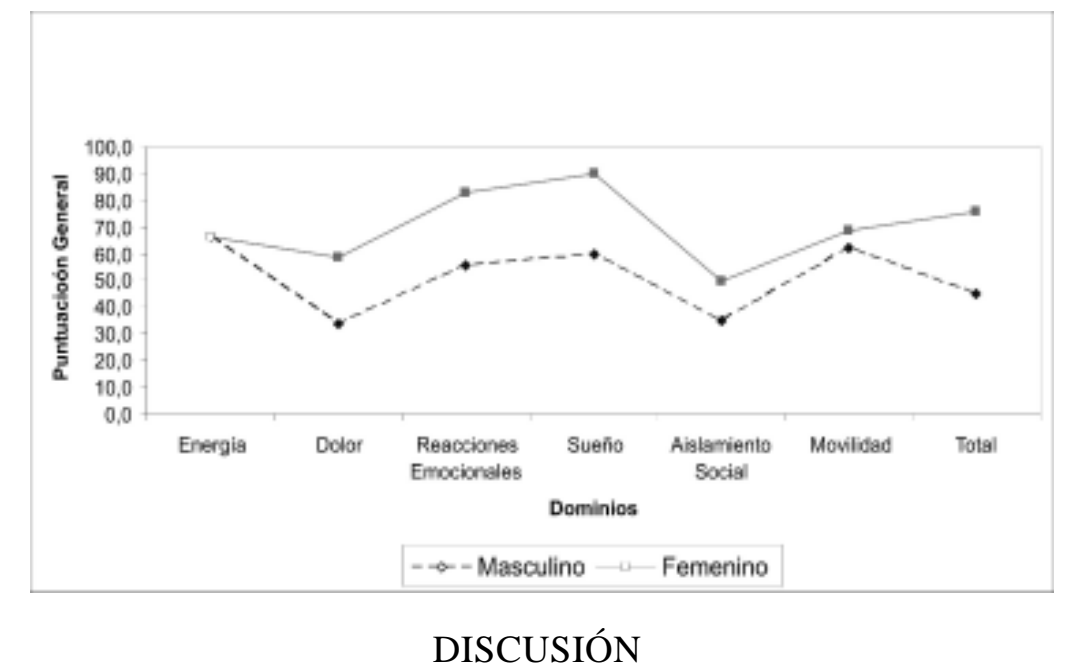

La muestra de pacientes tuvo una caracterización antropométrica y nutricional similar a los hallazgos encontrados en estudios de referencia (5-6-7). Sin embargo 
contrario a la literatura mundial la mayoría de nuestros pacientes fueron mujeres (63,6 \%), lo que no coincide con los resultados de otros estudios realizados en nuestro medio (16). Según los datos recogidos de la bibliografía, hasta un $25 \%$ de los pacientes portadores de EPOC presentan un estado de malnutrición el cual refleja un efecto claramente negativo en la evolución de la enfermedad, generando una mayor morbi mortalidad $(5-7,16)$. Basados en IMC, se pudo observar una estrecha variación entre los estados de desnutrición y eutrofismo. 10 pacientes (45\%), presentaron estado de eutrofia, (IMC 22 y $27 \mathrm{~kg} / \mathrm{m} 2$ ) mientras que los otros 12 pacientes (55\%), presentaron clasificaciones de desnutrición (IMC $<22$ kg/m2), según la clasificación propuesta por Harmonweiss (10). No se presentaron pacientes con grado de sobrepeso IMC $>27$ $\mathrm{kg} / \mathrm{m} 2$. Al analizar los resultados obtenidos con el NHP, las puntuaciones más altas (indicador de peor estado de salud) se reflejaron en los dominios Sueño, Reacciones Emocionales y Movilidad, coincidiendo con las demás investigaciones citadas en este trabajo (16-18). Al comparar los estudios que han utilizado el NHP en la bibliografía internacional con población semejante al nuestro, encontramos que Alonso (18) en 1992, demostró que las puntuaciones más altas fueron Energía, Movilidad y Sueño. Los resultados de Monso estableció considerables similitudes a este trabajo (19). Los resultados de Ferrer (20), coinciden con el nuestro ya que hallaron puntuaciones altas en todos los dominios. Sin embargo, nuestros pacientes acusaron un mayor deterioro en la calidad de vida. En último lugar, el apoyo social en EPOC, solo pudo ser contrastada con las cifras obtenidas en otros grupos de pacientes con otras patologías crónicas y solo un estudio con EPOC, utilizando los mismos instrumentos (17-20). Se observa que en nuestro caso fueron mayores, sobre todo los referidos en la escala de apoyo afectivo.

Limitaciones. Varios aspectos deben ser tenidos en cuenta como limitantes del estudio. En primer lugar, el tamaño de muestra y el tipo de muestreo puede introducir una serie de sesgos. En segundo lugar, la muestra no puede ser extrapolada como valor de referencia por no ser un grupo representativo de sujetos intervenidos

\section{REFERENCIAS}

1. Murray C, Lopez A. Mortality by cause for eight regions of the world: Global Burden of Disease Study. Lancet 1997;349:1269-1276.

2. Scharloo M, Kaptein AA, Schlösser M, Pouwels H, Bel EH, Rabe KF, Wouters EF. Illness perceptions and quality of life in patients with chronic obstructive pulmonary disease. J Asthma. 2007;44:575-581. 
REVISTADE SALUD PÚBLICA· Volumen 9 (4), Diciembre 2007

3. Mannino DM, Doherty DE, Sonia BA. Global Initiative on Obstructive Lung Disease (GOLD). Respir Med 2006;100:115-122

4. Ware J Jr. The status of health assessment 1994. Ann Rev Public Health 1995;16:327354.

5. Pauwels RA, Buist AS, Calverly PM, Jenkins CR, Hurd SS. Global strategy for the diagnosis, management, and prevention of chronic obstructive pulmonary disease. NHLBI/WHO Global Initiative for Chronic Obstructive Lung Disease (GOLD) Workshop summary, Am J Respir Crit Care Med 2001;163:1256-1276.

6. Barberá J, Peces-Barba G, Agustí A, Izquierdo J, Monsó E, Montemayor T, et al. Normativa SEPAR. Guía clínica para el diagnóstico y tratamiento de la enfermedad pulmonar obstructiva crónica. Arch Bronconeumol 2001;37:297-316.

7. Celli B, Snider G, Heffner J, Tiep B, Ziment I, Make B, et al. American Thoracic Society. Standards for the diagnosis and care of patients with chronic obstructive pulmonary disease. Am J Respir Crit Care Med 1995; 152 (Suppl): 77-120.

8. Mazza J, Carter J, Ross W, Ackiand T. Kinanthropometric Aquatic Sport Project. Aquatic Sport's World Champ. AUS. A proposal submitted to the VIII World FINAMedical Committee Meeting. London; 1991.

9. Esparza F. Manual de cineantropometría. Monografías Femede. $1^{\text {a }}$ ed. Navarra: Grupo Español de Cineantropometría(GREC); 1993.

10. Harmon-Weiss. Nutritional Strategies Efficacious in the prevention or treatment of Chronic Obstructive Disease Care. Washington 1999; 22-26.

11. Alonso J, Antó J, Moreno C. Spanish Version of the Nottingham Health Profile: translation and preliminary validity. Am J Public Health 1990; 80:704-708.

12. Alonso J, Prieto L, Antó JM. The Spanish version of the Nottingham Health Profile: a review of adaptation and instrument characteristics. Qual Life Res 1994; 3:385393.

13. Hunt S, Mckenna S, Mcewen J, William J, Papp E. The Nottingham Health profile: subjetive health status and medical consultations. Soc Sci Med 1981; 15:221229.

14. Bellón S, Delgado S, Luna del Castillo J. Validez y fiabilidad del cuestionario de apoyo social funcional DUKE-UNC-11. Aten Primaria 1996; 18:153-163.

15. Alvarado B, Zunzunegui M, Delisle H. Validation of food security and social support scales in an Afro-Colombian community: application on a prevalence study of nutritional status in children aged 6 to 18 months. Cad. Saúde Pública 2005: 21: 724-736.

16. Caballero A, Maldonado D, Torres C, Jaramillo C, Bermúdez M, Guevara D. Prevalencia de Síntomas Respiratorios de Colombia(PREPOCOL). Rev Col Neumol 2003, 15 (3):A166. 
17. Fernández V, Bujalance Z, Leiva F, Crespo J, García F. Sánchez C. Salud autopercibida, apoyo social y familiar de los pacientes con Enfermedad Pulmonar Obstructiva Crónica. MEDIFAM 2001;11:530-539.

18. Alonso J, Anto J, Gonzalez M, Fiz J, Izquierdo J, Morera J. Measurement of general health status of non-oxygen-dependent chronic obstructive pulmonary disease patients. Med Care 1992; 30(5 Suppl):MS125-MS135.

19. Monso E, FIZ J, Izquierdo J, Alonso J, Coll R, Rosell A, Morena J. Quality of life in severe Chronic Obstructive Pulmonary Disease (COPD): correlation whit lung and muscle function. Respire Ned 1998; 92:221-227.

20. Ferrer M, Alonso J, Morera J, Marrades R, Khalaf A, Aguar M, et al. Chronic Obstructive Pulmonary Disease Stage and Health-Related Quality of Life. Ann Intern Med 1997; 127: 1072-1079. 